\title{
Observational signatures of the interaction between acoustic waves and the solar magnetic canopy
}

\author{
P. F. Moretti ${ }^{1}$, S. M. Jefferies ${ }^{2}$, J. D. Armstrong ${ }^{2}$, and S. W. Mc Intosh ${ }^{3}$ \\ ${ }^{1}$ Istituto Tecnico Industriale Statale E. Fermi, via Trionfale 8737, Roma 00133, Italy \\ e-mail: pierfrancesco.moretti@uniroma1.it \\ 2 Institute for Astronomy, University of Hawai'i, 4761 Lower Kula Road, Kula, Hawaii 96790, USA \\ e-mail: stuartj@maile.ifa.hawaii.edu \\ 3 Department of Space Studies, Southwest Research Institute, 1050 Walnut Street, Suite 400, 80302 Boulder, CO, USA \\ e-mail: mcintosh@boulder.swri.edu
}

Received 6 February 2007 / Accepted 28 March 2007

\section{ABSTRACT}

\begin{abstract}
Aims. We show that the spatial distribution (and its variation with frequency) of the power spectra of the velocity and intensity signals, in and around solar active regions, is a manifestation of the interaction of acoustic waves at the magnetic canopy.

Methods. We analysed $6 \mathrm{~h}$ of simultaneous, full-disk, velocity and intensity images obtained using the MOTH instrument tuned in the $\mathrm{Na}$ D2 line at $589 \mathrm{~nm}$ and $\mathrm{K}$ D1 line at $770 \mathrm{~nm}$, and full-disk velocity images from the SOHO/MDI experiment using the Ni line at $677 \mathrm{~nm}$.

Results. We propose that more than one type of magneto-acoustic-gravity wave is required to explain the well-known phenomena of p-mode absorption and power halos.
\end{abstract}

Key words. Sun: atmosphere - Sun: oscillations - Sun magnetic fields

\section{Introduction}

Magneto-acoustic-gravity (MAG) waves are ubiquitous throughout the solar atmosphere. Their association with a wide range of phenomena, ranging from the heating of the atmosphere through to the generation of the solar wind, flares and coronal mass ejections, is testament to the importance of their role in the dynamics of the solar atmosphere and the Sun-Earth connection. Indeed, MAG waves can, in principle, be used for magneto-acoustic tomography of the solar atmosphere (Bogdan 2003) in much the same way as p-waves are used in local helioseismology to map the structure and dynamics immediately below the solar surface. Moreover, the potential for detailed atmospheric seismology is somewhat greater than it is for the interior due to the increased number of types of waves available (slow, fast and Alfvén). This being said, seismology of the atmosphere is in many ways more complex than it is for the interior. This is because there are as many as three types of waves that are moving information and energy through the atmosphere, each wave type contributing in a different way to the integrated intensity and Doppler velocity signals (Bogdan 2003). In addition, in regions where the gas and magnetic pressures are comparable (the magnetic canopy or $\beta \simeq 1$ region), the waves lose their identity and can readily transform from one type to another. This makes it challenging to monitor the propagation of the waves on their journey through the atmosphere (Bogdan \& Judge 2006). Indeed, numerical simulations of magneto-convection indicate that accurate deciphering of velocity and intensity observations at a given height in the solar atmosphere requires both knowledge of the location of the $\beta \simeq 1$ region with respect to the observing layer, and the connectivity of the magnetic flux that passes through the observing layer with the photosphere and or corona
(Bogdan \& Judge 2006). With this in mind, we have used a data set of imaged velocity and intensity signals, obtained simultaneously at three heights in the solar atmosphere, to study two phenomena whose origins are debated: the deficit of signal power in magnetic regions for waves with frequencies below the acoustic cut-off frequency $(\simeq 5 \mathrm{mHz})$, and the localized enhancement in the power around strong magnetic regions (hereafter referred to as halos) at frequencies above the cut-off (Braun et al. 1992; Toner \& LaBonte 1993; Hindman \& Brown 1998; Jain \& Haber 2002; Finsterle et al. 2004a).

\section{Observations}

We use approximately $6 \mathrm{~h}$ (from 2003.01.19 at 22:55:46 to 2003.01.20 at 04:36:56) of simultaneous, full-disk, velocity and intensity images obtained using the Magneto-Optical filters at Two Heigths (MOTH) instrument tuned in the Na D2 line at $589 \mathrm{~nm}$ and $\mathrm{K}$ D1 line at $770 \mathrm{~nm}$, and full-disk velocity images from the SOHO/MDI experiment using the Ni line at $677 \mathrm{~nm}$ (Scherrer et al. 1995). The MOTH observations were carried out from a site located some $6.3 \mathrm{~km}$ from the Amundsen Scott South Pole station, and the duty cycles of both data sets are close to $100 \%$. The temporal and spatial resolutions of the MOTH and MDI data sets are $10 \mathrm{~s} / 10$ arcsec and $60 \mathrm{~s} / 4$ arcsec, respectively (Finsterle et al. 2004a). After registration and remapping to a common grid, an active region was tracked throughout its passage across the disk. The power spectra for each pixel were then computed and frequency filtered using a boxcar of width $0.6 \mathrm{mHz}$ to yield the final power map. Maps of wave travel time between the different observing layers were also computed as described in Finsterle et al. (2004b). The interpretation of the observations requires knowledge of the location of the magnetic 

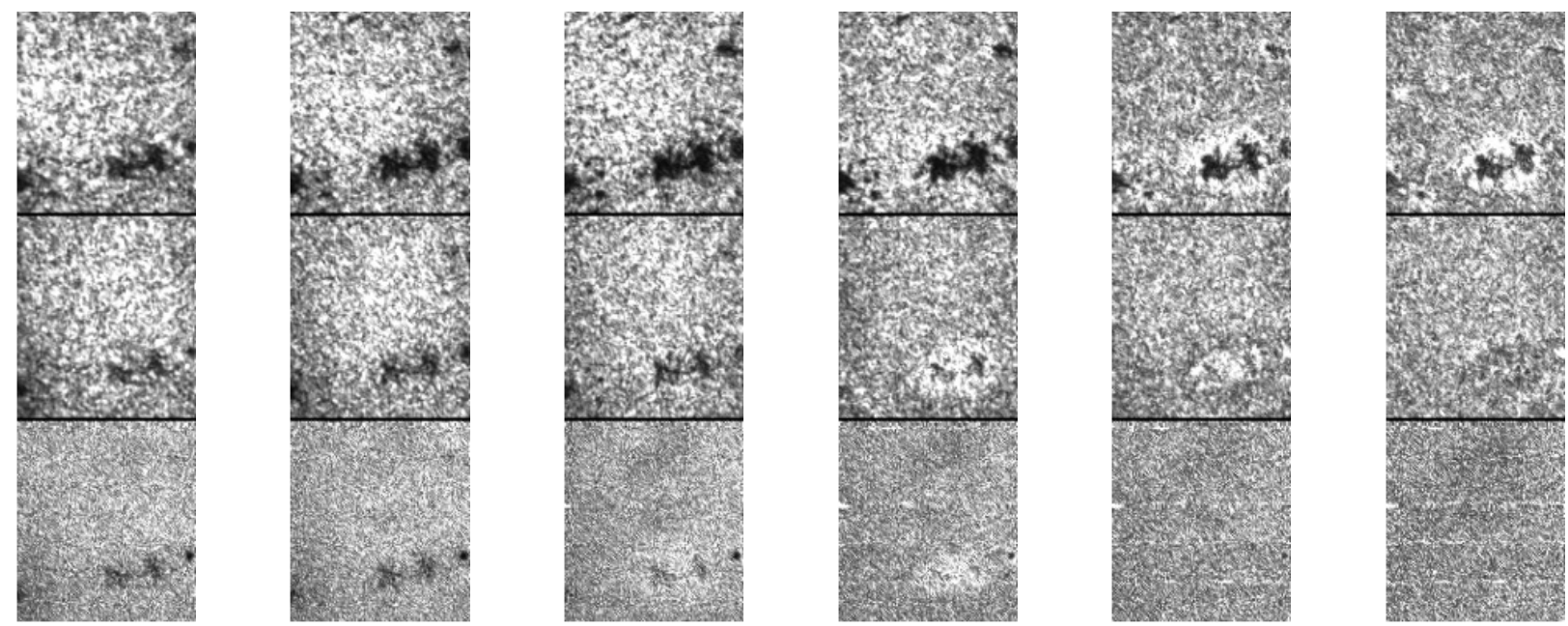

Fig. 1. Velocity power maps at 3,4,5,6,7 and $8 \mathrm{mHz}$ (from left to right) based on approximately $6 \mathrm{~h}$ of disk center solar images. Data were taken on January 20, 2003 with the MOTH experiment running at South Pole and tuned in the Na $590 \mathrm{~nm}$ solar line (top panels) and in the K $770 \mathrm{~nm}$ solar line (central panels). Simultaneous data were obtained from the MDI/SOHO experiment in the Ni $677 \mathrm{~nm}$ solar line (bottom panels). The mean heights for the formation layers for the $\mathrm{Na}, \mathrm{K}$ and $\mathrm{Ni}$ observations are $500 \mathrm{~km}, 250 \mathrm{~km}$ and $100 \mathrm{~km}$ respectively (see text). The power maps are integrated over the frequency range using a boxcar of width $0.6 \mathrm{mHz}$. The maps correspond to a region of -20 degrees to 20 degrees both in latitude and longitude. Longitude 0 crosses disk center on 2003.01.20 at 09:25:56. Top is North, left is West.

canopy through the chromosphere. We estimate the magnetic field in the chromosphere using a potential field extrapolation of contemporaneous SOHO/MDI observations of the line-of-sight magnetic field. This is then used in conjunction with values for the gas pressure from the the FAL 93 atmosphere models " $\mathrm{C}, \mathrm{F}$ and $\mathrm{P}$ " representing the different components observed in quiet Sun (Fontela et al. 1993), to generate maps of the $\beta$ surfaces from 0 to $3000 \mathrm{~km}$ (Finsterle et al. 2004b). A limitation of our magnetic field reconstruction, as well as the potential magnetic field extrapolation based on the solely longitudinal magnetic flux maps, is that it can not quantify accurately the field inclinations.

To compare the $\beta \simeq 1$ maps with observations requires knowledge of the heights of formation of our lines. Formation heights are commonly quoted for models that treat the solar atmosphere as being static. However, the solar atmosphere is highly dynamic and the real heights of formation are likely to be substantially different from the values based on static models. Indeed, three-dimensional hydrodynamic simulations of solar convection show that for a non-magnetic atmosphere the effective formation heights of the $\mathrm{Na}(589 \mathrm{~nm})$ and $\mathrm{K}(770 \mathrm{~nm})$ lines (Uitenbroek 2006), as sampled by the MOTH filters, can be expected to range between $200-800 \mathrm{~km}$ for $\mathrm{Na}$ and $50-450 \mathrm{~km}$ for $\mathrm{K}$. We therefore ascribe mean heights for our $\mathrm{Na}$ and $\mathrm{K} \mathrm{ob}-$ servations of regions of magnetically quiet Sun, of $\sim 500 \mathrm{~km}$ and $\sim 250 \mathrm{~km}$, respectively. Using a similar approach for the Ni line data observed by MDI we estimate the mean height for the Ni observations of $100 \mathrm{~km}$. We note that the large uncertainty in these values translates into large uncertainties in the estimated density values used below (factor of 5 or more). The uncertainty is further compounded by our lack of understanding of how the different plasma properties of a magnetic atmosphere affect the $\mathrm{Ni}, \mathrm{K}$, and $\mathrm{Na}$ response functions for the line-of-sight velocity, in particular, whether there are relative shifts between them that are different in magnetic and non-magnetic plasmas (Jefferies et al. 2006).

The main observational results are:

a) The velocity power maps for frequencies below the cut-off frequency show that the spatial distribution of the power deficits in the regions of the strongest magnetic field depends on height (Fig. 1). This is evident looking at the power of the magnetic region in the top right of the panels in Fig. 1. This magnetic region is essentially invisible at the lowest height (the Ni maps) while the deficit in the power is present in the upper layers with different traits.

b) The velocity power maps for frequencies above the cut-off frequency show that the spatial distribution of the regions of enhanced power changes with frequency and height. The power halos are present over a limited frequency range that depends on the height of the observations. Halos fill in both between the bipolar active region and in the active region where a power deficit is observed at low frequencies: this is evident in the $\mathrm{Ni}$ and $\mathrm{K}$ power maps (Fig. 1), while in the $\mathrm{Na}$ maps such filling is visible only in comparison with the low frequency maps (Fig. 2). Since a power deficit is observed again at very high frequencies (see Fig. 4), the filling of power is only present in a limited range of frequency.

To study this further we selected the most powerful pixels (larger that 1.66 times the mean value over the whole field of view) in the vicinity of the active region and obtained a mask by summing the occurrence of these locations from 5 to $10 \mathrm{mHz}$. The ratio between the power integrated over this mask and that integrated over the same mask shifted in a quiet sun region shows that the enhancement of the power is peaked at different frequencies for the different heights (see Fig. 5). A similar behavior was previously reported in the $\mathrm{Ni}$ data by Hindman \& Brown (1998) but the mask was obtained using the magnetic field strength as a selective filter. Since the visibility of the halos has been demonstrated to be independent of magnetic field strength (Hindman \& Brown 1998; Braun \& Lindsey 1999), the halos signal obtained using this latter approach has a component that comes from magnetic regions far from the active regions under study. This has the effect of shifting the peak of the ratio of the magnetic to nonmagnetic signal to higher frequencies than are indicated in Fig. 5, where we measure peak frequencies for the visibility of the halos for the $\mathrm{Ni}, \mathrm{K}$ and $\mathrm{Na}$ observations at approximately 5.5, 6.0 and $7.5 \mathrm{mHz}$, respectively. 

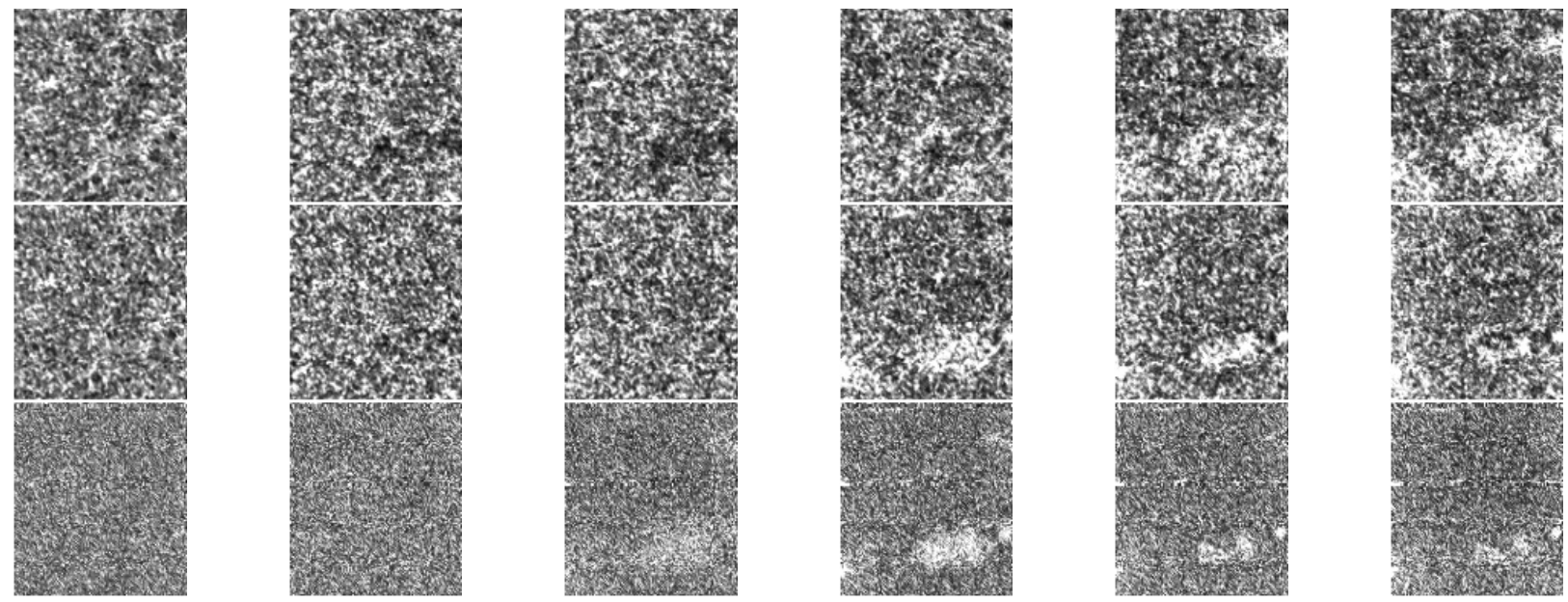

Fig. 2. The velocity power maps shown in Fig. 1 but divided by the map at $3.3 \mathrm{mHz}$ corresponding to each layer.
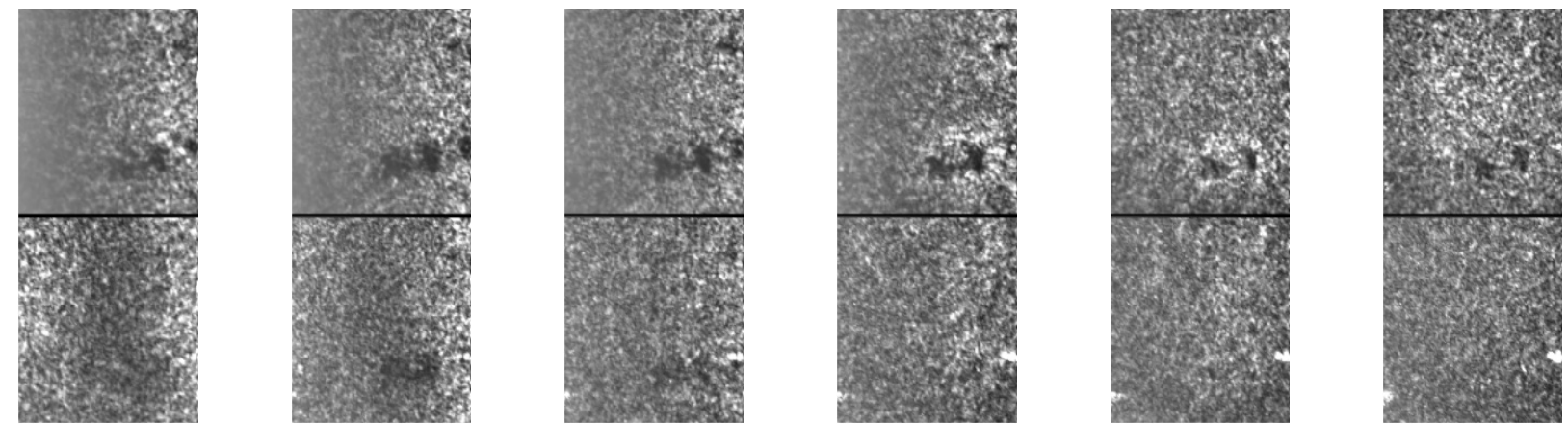

Fig. 3. Intensity power maps 3, 4, 5, 6, 7 and $8 \mathrm{mHz}$ (from left to right) of the MOTH data shown in Fig. 1 (Na and $\mathrm{K}$ observations in top and bottom panels respectively).

c) Intensity halos are visible in the Na observations. They are also visible at the $\mathrm{K}$ formation layer when an integration over the halo positions, obtained in the same way as for the velocity power maps, is performed (Figs. 3 and 5). Because of the inherent properties of the observations obtained with twopoint filters, such as those employed by the MOTH experiment, we have to consider the possibility of a crosstalk between the intensity and velocity signals (Moretti \& Severino 2004). The comparison of the ratios of the intensity halos at different frequencies does not reproduces those obtained for the velocity. Intensity maps would reproduce the velocity maps in case of complete instrumental crosstalk or in case of compressive waves observed along the propagation direction. In our case, the traits of the intensity halos show that the instrumental crosstalk is negligible and that the compressive waves we observed were propagating mainly not along the line of sight.

d) Maps of the time taken for the high-frequency propagating waves to travel between the $\mathrm{K}$ and $\mathrm{Na}$ heights of formation show that downward propagating waves are only detected in the active regions where there is absorption at $3 \mathrm{mHz}$ and power enhancement above $5 \mathrm{mhz}$ (see Fig. 6).

\section{Discussion}

Mode conversion is the most likely candidate for describing the power deficit in strong magnetic regions (Cally et al. 2003). When high $-\beta$ waves traveling from the interior meet the $\beta \simeq 1$ region, they are partially converted to high $-\beta$ slow waves which, because of their transverse nature and the lack of any inclination in the local field lines, are not detected in either the line-ofsight velocity or intensity observations. At low frequencies (below $\simeq 5 \mathrm{mHz}$ ), where the waves should be evanescent, the deficit of the power is therefore expected to be visible only where the $\beta \simeq 1$ region penetrates down into the photosphere. In this case, the deficit is also expected to be independent on height. Indeed, the different spatial distributions of the deficits at low fequencies (topic (a) in the observations paragraph) suggests that the waves are not evanescent. In fact, it has been recently shown that the cut-off frequency depends on the inclination of the magnetic field lines. As a result, in magnetic regions energy can propagate upwards into the overlaying atmosphere at lower frequencies (Bel \& Leroy 1977; Schunker \& Cally 2006; Jefferies et al. 2006; McIntosh \& Jefferies 2006) and the propagated energy is peaked around an inclination angle different from the vertical (Schunker \& Cally 2006). Therefore, the spatial distribution of the power deficit describes the condition where the height at which an upcoming acoustic wave of frequecy $v$ reflect downward is higher than the magnetic canopy. That is, we expect the $\beta \simeq 1$ contours at the observation height to outline the regions of power deficit.

In summary, high-frequency waves and low-frequency waves where the magnetic lines are inclined, are sensitive to the location of the magnetic canopy (Finsterle et al. 2004a; Schunker \& Cally 2006). It is also demonstrated both in Fig. 1 and Fig. 7 , where a region shows a power deficit only in the Na observations because the magnetic canopy is formed above the height of the K observations but below the height of the Na observations. 


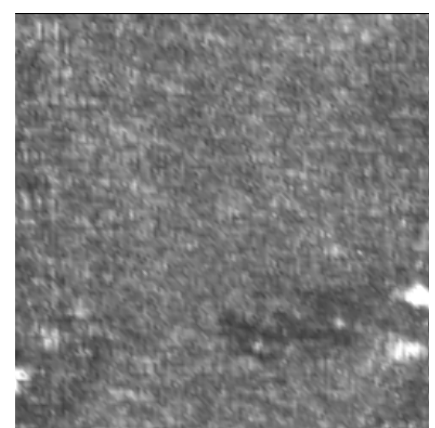

Fig. 4. Velocity power map of $\mathrm{K}$ observations at $20 \mathrm{mHz}$. The power map is integrated over the frequency range using a boxcar of width $3 \mathrm{mHz}$.
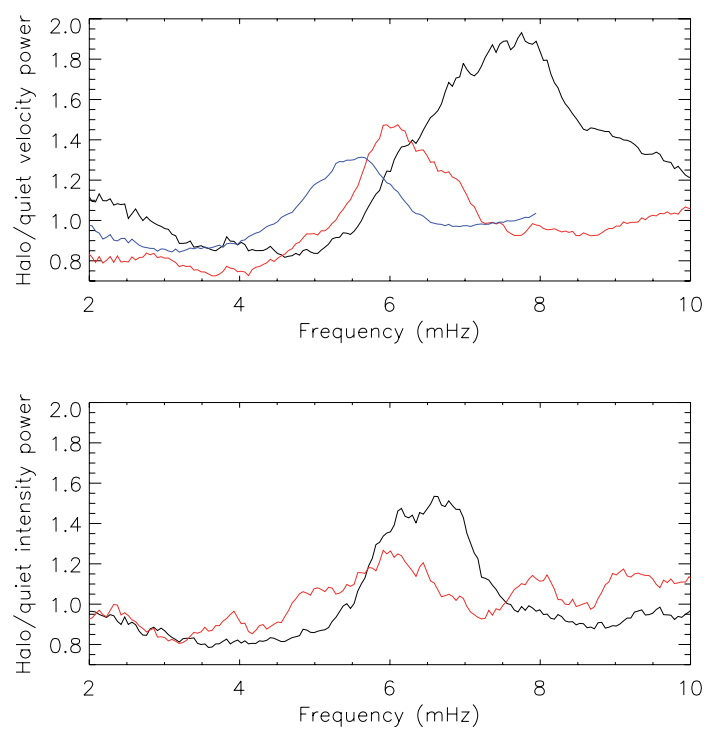

Fig. 5. Visibility of the halos in velocity (top) and intensity (bottom) for $\mathrm{Na}$ (black), K (red) and Ni (blue). At each frequency, the most powerful pixels (larger that 1.66 times the mean value over the whole field of view) in the vicinity of the active region were selected and a mask made by summing the occurrence of these locations from 5 to $10 \mathrm{mHz}$. The ratios between the power integrated over this mask and that integrated over the same mask shifted in a quiet sun region are shown.

Explanation of the remaining observations (b-d requires the presence of three types of MAG waves (see sketch in Fig. 8). When the frequency of a high- $\beta$ fast wave impinging on the $\beta \simeq 1$ region matches the Alfvén frequency for the local magnetic field, the amplitudes of the waves emerging from the magnetic canopy are dramatically increased (due to resonance). In this case, any wave at the resonant frequency can be assumed to dominate those at different frequencies. Such resonant behavior can explain both the peak visibility and its variation in frequency with changing height. The latter is because, for a fixed wavelength, the decreasing density and slowly changing magnetic field strength with increasing height requires a higher frequency for resonance. The wavelength is related to the length of the magnetic field line. If the field lines at the different heights have similar geometries, then the ratio of the wavelengths can be assumed to be given by the ratio of the heights, and we have $R\left(z_{1}: z_{2}\right)=v\left(z_{2}\right) / v\left(z_{1}\right)=\left(z_{1} / z_{2}\right) \sqrt{\rho\left(z_{1}\right) / \rho\left(z_{2}\right)}$. When $v(z)$ is the peak frequency at the height $z$ and $\rho$ is the density. Using the densities at 200, 350 and $550 \mathrm{~km}$ from Vernazza et al. (1981), that is $8.4 \times 10^{-8}, 2.5 \times 10^{-8}, 3 \times 10^{-9} \mathrm{~g} \mathrm{~cm}^{-3}$ respectively, we obtain $R(\mathrm{Na}: \mathrm{Ni})=1.9, R(\mathrm{Na}: \mathrm{K})=1.8$. These values are approximately $30 \%$ higher than the observed values. Considering the relatively
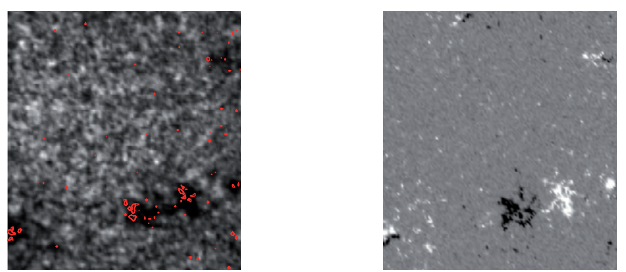

Fig. 6. Left panel: velocity power map at $3 \mathrm{mHz}$ for the $\mathrm{Na}$ observations, superimposed with the contours of the phase travel times corresponding to downward propagating waves at $7 \mathrm{mHz}$ (in red). The downward waves are located mainly where a power deficit is observed at low frequencies. Right panel: the corresponding line-of-sight magnetic field maps obtained from the MDI/SOHO experiment. Black to white scale from 200 to -200 Gauss.
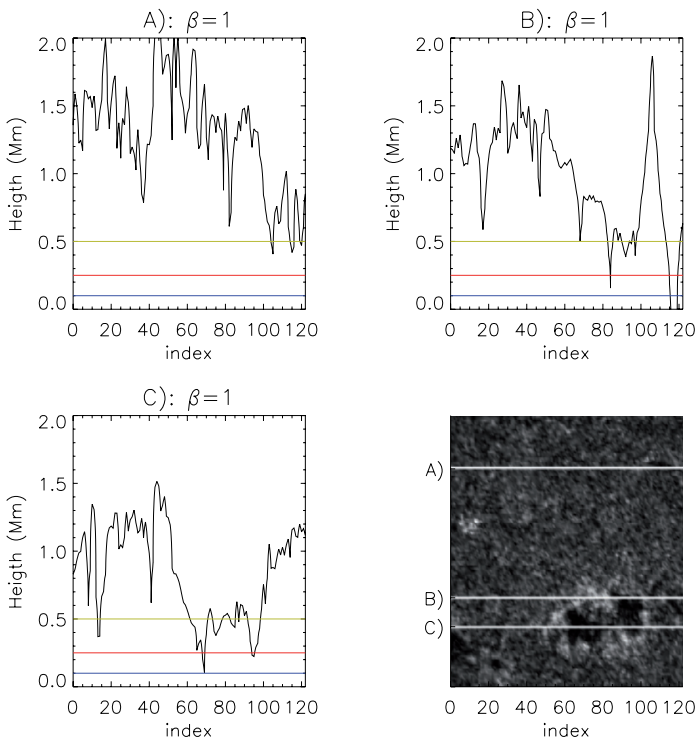

Fig. 7. Panels a), b) and c) show the profiles of the $\beta=1$ contours along the rows a), b) and c) in the $7 \mathrm{mHz}$ velocity power map for the Na observations shown in the bottom right panel.

large uncertainties in the heights of formation and the densities, this can be considered a good match.

If the efficiency with which the high- $\beta$ fast waves are converted into each of the wave types is sensitive to the inclination of the magnetic field with respect to the wave vector (the impact angle), then the efficiency of conversion to transverse waves will increase as the impact angle increases. At high impact angles, transverse waves dominate. Because a large fraction of the magnetic field at low heights can be highly inclined, on the high- $\beta$ side of the magnetic canopy the observed signal is a combination of that from Alfvén waves and interference of upward and reflected high- $\beta$ fast waves. On the low- $\beta$ side, the observed signal is a combination of all three types: Alfvén, slow and fast. The low- $\beta$ slow waves are longitudinal and guided along the magnetic lines, that is, detectable only when the magnetic field has a significant line-of-sight projection. At disk center, we expect to observe their signature only at locations of the strong magnetic field. This is confirmed by the increased power observed at high frequencies in strong magnetic regions where a power deficit is observed in the low frequency range. When wave conversion occurs above the upper observing layer, low- $\beta$ field guided slow waves should appear as downward traveling waves between the two observing heights. The observed phase travel time maps indeed show downward propagating waves that are located only inside the low- $\beta$ side of the magnetic canopy. 


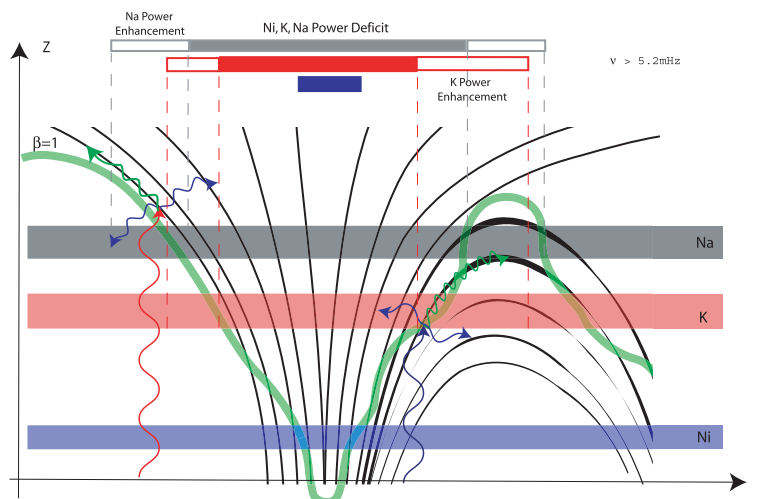

Fig. 8. Sketch of the interaction of acoustic waves with the magnetic canopy. The solid line represents a typical $\beta \simeq 1$ contour between the bipolar region as shown in Fig. 7. The dashed lines represent schematic magnetic field lines. The blue, red and grey regions represent the formation layers for the $\mathrm{Ni}, \mathrm{K}$ and $\mathrm{Na}$ observations respectively. On the left: the observed deficit in power depends on the inclination of the magnetic lines (influencing the cut-off frequency and the propagation of the waves through the upper layers) and where the $\beta \simeq 1$ region is placed.

The filling of the velocity power in between the bipolar active region is due to a combination of Alfvén waves traveling along field lines that are close to horizontal at the observation heights plus interference of high- $\beta$ fast waves. These require high impact angles and the $\beta \simeq 1$ region above and close to the formation height. This second requirement is not met by our sodium observations because, for this particular data set, the magnetic canopy in between the bipolar active region is lower than the formation height (see Fig. 7).

At low impact angles, the generation of transverse waves is reduced with respect to the other types of waves emerging from the $\beta \simeq 1$ region. In the high- $\beta$ side of the magnetic canopy, the signal mainly consists of fast waves, both upward and reflected, while in the low- $\beta$ side, slow waves are mainly present. On the outskirts of the bipolar region the structure of the magnetic filed lines is such that the average impact angle decreases with increasing height, and both velocity and intensity halos surrounding bipolar acive regions are observed in the sodium layer.

The intensity oscillations reflect the compressional component of the waves. The intensity halos are difficult to see in the absorption regions because of the reduced dynamic range and this could be the reason why they were not observed in the MDI observations (Hindman \& Brown 1998; Jain \& Haber 2002).

\section{Conclusions}

The magnetic canopy has been recently invoked to explain the power deficit in, and halos around, active regions (Cally et al. 2003; Muglach et al. 2005). Our results support this general picture. However, because previous analyses have integrated over wide frequency intervals when studying these phenomena, several crucial traits were hidden. We show that explanation of these traits, in particular those associated with the halos, requires the presence of more than one type of MAG wave. This confirms the results from numerical models that suggest that the wavefiled in the solar atmosphere is highly complex. It is clear that the future for tomography of the solar atmosphere lies in the synthesis of simultaneous, high-resolution, multi-observable (velocity, intensity and magnetic field), multi-height observations and large scale numerical modeling of magneto-convection.

Acknowledgements. We thank Han Uitenbroek for the calculations of the contribution functions for the heights of formations. This work utilizes data obtained by the 2002-2003 Magneto-Optical filter at Two Heights (MOTH) project: a collaboration between the University of New Mexico, USA and the University of Rome, Italy. The MOTH project was supported by the National Science Foundation under award numbers ANT-0087541 \& ANT-0632399. P.F.M. thanks Federica Brandizzi.

\section{References}

Bel, N., \& Leroy, B. 1977, A\&A, 55, 239

Bogdan, T. J., \& Judge, P. G. 2006, R. Soc. London Tran. Ser. A, 364, 313 Bogdan, T. J., Carlsson, M., Hansteen, V. H., et al. 2003, ApJ, 599, 626

Braun, D. C., \& Lindsey, C. 1999, ApJ, 513, L79

Braun, D. C., Lindsey, C., Fan, Y., \& Jefferies, S. M. 1992, ApJ, 392, 739

Cally, P. S., Crouch, A. D., \& Braun, D. C. 2003, MNRAS, 346, 381

Hindman, B. W., \& Brown, T. M. 1998, ApJ, 504, 1029

Finsterle, W., Jefferies, S. M., Cacciani, A., et al. 2004a, Sol. Phys., 220, 317

Finsterle, W., Jefferies, S. M., Cacciani, A., Rapex, P., \& McIntosh, S. W. 2004b, ApJ, 613, 185

Fontenla, J. M., Avrett, E. H., \& Loeser, R. 1993, ApJ, 406, 319

Jefferies, S. M., McIntosh, S. W., Armstrong, J. D., et al. 2006, ApJ, 648, L151

McIntosh, S. W., \& Jefferies, S. M. 2006, ApJ, 647, L77

Jain, R., \& Haber, D. 2002, A\&A, 387, 1092

Moretti, P. F., \& Severino, G. 2004, A\&A, 421, 729

Muglach, K., Hofmann, A., \& Staude, J. 2005, A\&A, 437, 1055

Schunker, H., \& Cally, P. S. 2006, MNRAS, in press

Scherrer, P. H., Bogart, R. S., Bush, R. I., et al. 1995, Sol. Phys., 162, 129

Toner, C. G., \& LaBonte, B. J. 1993, ApJ, 415, 847

Uitenbroek, H. 2006 in Solar MHD: Theory and Observations - a High Spatial Resolution Perspective, ed. R. L. Stein, J. Leibacher, \& H. Uitenbroek (NSO Workshop 23; Sunspot: NSO), in press

Vernazza, J. E., Avrett, E. H., \& Loeser, R. 1981, ApJS, 45, 635 